\title{
Estimación probabilística del volumen de acarreos en suspensión en cuencas sin aforos de la Región Hidrológica No. 10 (Sinaloa)
}

\section{Probabilistic Estimation of Suspenden Sediment Volume in Watersheds Without Hydrometric Data of the Hydrological Region No. 10 (Sinaloa)}

\author{
D.F. Campos-Aranda \\ Facultad de Ingeniería. \\ Universidad Autónoma de San Luis Potosí. México. \\ E-mail:campos_aranda@hotmail.com
}

(Recibido: junio de 2007, aceptado: septiembre de 2008)

\section{Resumen}

Inicialmente se proponen tres acciones para contrarrestar los efectos negativos del cambio climático en los diseños hidrológicos. Además, se destaca la utilidad del análisis regional de datos hidrológicos para obtener procedimientos y fórmulas de estimación de valores de diseño más confiables en cuencas sin aforos. En seguida se describe la información de acarreos en suspensión disponible en la Región Hidrológica No. 10 (Sinaloa) y se cita la que fue utilizada. Se acepta la homogeneidad de la región con base en la relación encontrada entre el volumen de sedimentos promedio anual y el área de cuenca. Posteriormente se describen y aplican tres métodos regionales: (1) el de las estaciones-año, (2) el de los momentos de probabilidad pesada (MPP) regionales y (3) el de los MPP ponderados. Se continúa con la aplicación numérica relativa a la estimación de la capacidad de azolves en tres embalses de la región. Por último, se formulan las conclusiones las cuales destacan las ventajas del enfoque propuesto

Descriptores: Sedimentos, análisis regional, momentos de probabilidad pesada, modelo GVE.

\begin{abstract}
Firstly three actions are proposed to mitigate the negative effects of climatic change in the hydrological design. Besides is pointed out the utility of regional analysis of hydrological data to obtain more reliable formulae and procedures for estimating design values in watersheds without hydrometric data. Next, the suspended sediment data available in Hydrological Region number 10 (Sinaloa) is described and the ut $i-$ lized data is cited. The homogeneity of that region was accepted through the relation between watershed area and mean annual suspended sediment volume. Late three regional methods are described and applied, these are: (1) the station-year method, (2) the regional probability weighted moments ( $P W M$ ) method and (3) the weighted PWM method. Next, the numerical application for sediment capacity estimation in three reservoirs of the region is described. Lastly conclusions are formulated which pointed out the advantages of the proposal approach.
\end{abstract}

Keywords: Sediment, regional analysis, probability weighted moments, GVE distribution. 
DOI: http://dx.doi.org/10.22201/fi.25940732e.2009.10n3.021

Estimación probabilística del volumen de acarreos en suspensión en cuencas sin aforos de la Región Hidrológica No. 10 (Sinaloa)

\section{Introducción}

El cambio climático inminente está generando condiciones meteorológicas más extremas, concretamente tormentas más severas y sequías más prolongadas. Ambas condiciones producirán seguramente, magnitudes más elevadas de la erosión de los suelos, debido al mayor poder erosivo de la lluvia y a la menor densidad de cobertura vegetal. En realidad, el poder erosivo de las tormentas severas y la capacidad de transporte de las crecientes que originan, es conocido desde hace décadas, cuando se observó que éstas, en pocas horas, pueden aportar más sedimento que el ocurrido en varios años. Ya que el sedimento transportado por los cauces, es el responsable de la pérdida de almacenamiento de los embalses por sedimentación; surge entonces una pregunta fundamental ¿Cómo mejorar la estimación del volumen destinado a la acumulación de los sedimentos en los embalses o capacidad de azolves frente al cambio climático?

En términos generales, ante el cambio climático se deben realizar las siguientes tres acciones: primera, por ningún motivo suspender mediciones hidrométricas y climatológicas, ya que ahora resulta fundamental incorporar en ellos los nuevos valores extremos, de manera que las actualizaciones de los estudios hidrológicos reflejen verdaderamente las nuevas condiciones o tendencias climáticas. Segunda, debido a que las condiciones meteorológicas están cambiando y por consecuencia las climáticas, actualmente es más imperativo ampliar los puntos de medición, para disponer de valores de apoyo reales en el transporte de información de sitios con registros amplios a estas nuevas localidades con escasez de datos, así como para realizar en el futuro análisis regionales más confiables.

La tercera acción para contrarrestar los efectos negativos del cambio climático, consiste en aumentar el grado de confiabilidad de las estimaciones hidrológicas, a través de incrementar el nivel de probabilidad utilizado. Por ejemplo, en lugar de utilizar la mediana del volumen anual de sedimentos, o estimación con un $50 \%$ de probabilidad de excedencia, ya que en uno de cada dos años tal valor puede ser excedido, se debe emplear el valor que sólo es excedido una vez en tres, cuatro o cinco años, esto es, emplear magnitudes de diseño con probabilidades de no excedencia del 66.7, 75 y $80 \%$, es decir, pedir que en cada 3, 4 ó 5 años únicamente uno presente un valor igual o mayor.

Por otra parte, el análisis regional de datos busca relaciones generales y confiables válidas dentro de una región o zona geográfica homogénea, entre la variable de interés en el diseño hidrológico y otra u otras fácilmente accesibles (Cunnane, 1988). Lógicamente, la utilidad de las relaciones encontradas radica en permitir diseños hidrológicos menos empíricos en cuencas sin aforos.

El análisis regional de datos anuales de acarreos en suspensión que se desarrolla en este trabajo, está orientado a la estimación de los valores de diseño en cuencas sin tales registros, los cuales están asociados a las probabilidades de no excedencia citadas. Por lo anterior, el planteamiento general comprende: (1) la descripción general de la información disponible, así como la selección y presentación de la que será procesada; (2) el desarrollo de los procedimientos regionales que son aplicables y que conducen a las predicciones buscadas y (3) la descripción y contraste de unas aplicaciones prácticas.

\section{Desarrollo}

\section{Datos disponibles}

El transporte o carga de sedimentos de una corriente consta de dos tipos definidos con base en el tamaño de las partículas y la forma de movimiento; el primero se denomina sedimento en suspensión, que es el material fino mantenido en el tirante o lámina de agua por efecto de la turbulencia de la corriente o en suspensión coloidal. El segundo se conoce como transporte de fondo, es el material grueso que se mueve continuamente o de manera intermitente por rodamiento o saltación. Generalmente, varía del 5 al 35\% del transporte en suspensión (Morris y Fan, 1998).

De acuerdo al Boletín Hidrológico No. 36 (SRH, 1975) de la Región Hidrológica No. 10 (Sinaloa), se dispone de un total de 17 estaciones hidrométricas que cuentan con información denominada de acarreos en suspensión, expresados en miles de toneladas y de $\mathrm{m}^{3}$. Seis de tales estaciones fueron operadas por la Comisión Federal de Electricidad (CFE) y no se dispone de datos en tres de ellas (La Veranera, Guerachic y Tecusiapa). La estación San Francisco fue operada por la CFE y sus 27 datos anuales utilizados proceden del Tomo VI del boletín citado. Para las trece estaciones restantes, los datos están disponibles en el sistema BANDAS (IMTA, 2003), del cual se recopiló la información general y relativa a sus valores anuales, con tales datos se integró la tabla 1. En total se dispuso de 281 valores anuales.

\section{Datos utilizados y homogeneidad regional}

En la tabla 1 se observa que cuatro estaciones hidrométricas tienen registros demasiado cortos menores de 9 años, que en total suman sólo 27 valores anuales, por 
DOI: http://dx.doi.org/10.22201/fi.25940732e.2009.10n3.021

D.F. Campos-Aranda

ello, se decidió prescindir de tales registros. Con respecto a las estaciones de registro corto ( $\leq 15$ años), Choix y La Huerta, se decidió eliminar a la segunda, pues su registro corresponde a un periodo de únicamente años secos. Respecto al registro de la estación hidrométrica $\mathrm{Pa}-$ los Blancos, éste abarca hasta 1962, porque en 1963 empezó a operar aguas arriba la presa Adolfo López Mateos (Humaya).

El resto de sólo nueve estaciones hidrométricas con un total 239 valores anuales, los cuales se muestran en la tabla 2, constituye una disponibilidad escasa para realizar un análisis de homogeneidad tendiente a definir subregiones, por lo cual se consideró homogénea la Región Hidrológica No. 10.

Esta región ha sido encontrada homogénea con base en pruebas estadísticas aplicadas a gastos máximos anuales por Campos (1994) y Domínguez (1996). En cambio, Escalante (1998) utilizando el método de las curvas multidimensionales, definió dos subregiones una al Norte integrada por las cuencas de los ríos Fuerte y Sinaloa, la otra al Sur abarca el resto de la región hasta el río Quelite.

En la tabla 3 la llamada aportación de sedimentos media anual $(A S)$ varía de 202.6 a $479.8 \mathrm{~m}^{3} / \mathrm{km}^{2}$, este intervalo es bastante reducido de acuerdo a las magnitudes citadas en la literatura especializada (Morris y Fan, 1998) y con ello, se genera cierta confianza con respecto a la homogeneidad de la Región Hidrológica No. 10, en cuanto a volúmenes de acarreos en suspensión. También en la tabla 3 se presentan los principales parámetros físicos de las cuencas de las nueve estaciones hidrométricas que serán utilizadas, tomados de Escalante y Reyes (2002).

\section{Estimación del VSMA}

Al dibujar en un papel logarítmico en las abscisas las áreas de cuenca en $\mathrm{km}^{2}$ y en las ordenadas los volúmenes de sedimento medio anual en miles de $\mathrm{m}^{3}$ (figura 1), es decir, las columnas 4 y 3 de la tabla 3 , se observa que tales datos definen una relación lineal expresada por la ecuación:

$$
V S M A=0.20186 \cdot A^{1.05243}
$$

con un coeficiente de determinación $\left(\mathrm{R}^{2}\right)$ de 0.947 y un error estándar de la estimación (EEE) de 667.8 miles de $\mathrm{m}^{3}$. Con base en la información de la tabla 3 se pueden plantear diversas regresiones lineales múltiples como las que se citan a continuación:

$V S M A=709.2326+0.34101 \cdot A-0.81763 \cdot P M A$ con

$$
R^{2}=0.941 \quad E E E=828.6 \text { miles de } \mathrm{m}^{3}
$$

$V S M A=1,937.521+0.3085 \cdot A-2.80 .8 P M A+22,338.9 \cdot S c$

con

$$
R^{2}=0.944 \quad E E E=881.6 \text { miles de } \mathrm{m}^{3} .
$$

Como se observa, no se logra mejorar estadísticamente la estimación del VSMA, por lo cual se empleará la ecuación 1 como relación funcional de la Región Hidrológica No. 10, la cual además verifica la homogeneidad hidrológica de ésta (figura 1).

\section{Método de las estaciones-año}

Su objetivo consiste en lograr un mejor ajuste de un modelo probabilístico y sobre todo, unos resultados más confiables al utilizar un registro mucho más amplio, el constituido por todos los valores anuales de las estaciones que integran la zona o región hidrológicamente homogénea. Para suprimir el efecto de escala de cada una de las cuencas, sus datos anuales se estandarizan dividiéndolos entre su media aritmética, por lo anterior 1.000 será la media de la serie integrada.

El ajuste por momentos L de la distribución GVE (Stedinger et al., 1993; Campos, 2006) a la serie integrada, condujo a sus siguientes parámetros: $u=0.62378, a=0.41037 \mathrm{y} k=-0.26366$, con las predicciones $(X)$ adimensionales mostradas en la tabla 4.

El valor de $X$ al ser multiplicado por el VSMA estimado con la ecuación 1 conducirá a las magnitudes de diseño.

\section{Método de los MPP regionales}

Con base en los resultados de Varas (2000) y de Campos (2005), es posible el ajuste regional de la distribución GVE mediante momentos L en cuencas sin datos hidrométricos, ya que los momentos de probabilidad pesada (MPP) $b_{1}$ y $b_{2}$, pueden ser estimados con base en el $b_{0} \mathrm{O}$ media aritmética, como se indica a continuación. Se comienza por ordenar en forma decreciente los datos $x_{i}$ $\left(x_{1}>x_{2}, x_{2}>x_{3}, \ldots, x_{n-1}>x_{n}\right)$, después a partir de las expresiones siguientes se calculan los MPP insesgados $b_{i}$ (Stedinger et al., 1993), cuyos valores se presentan en la tabla 5. 


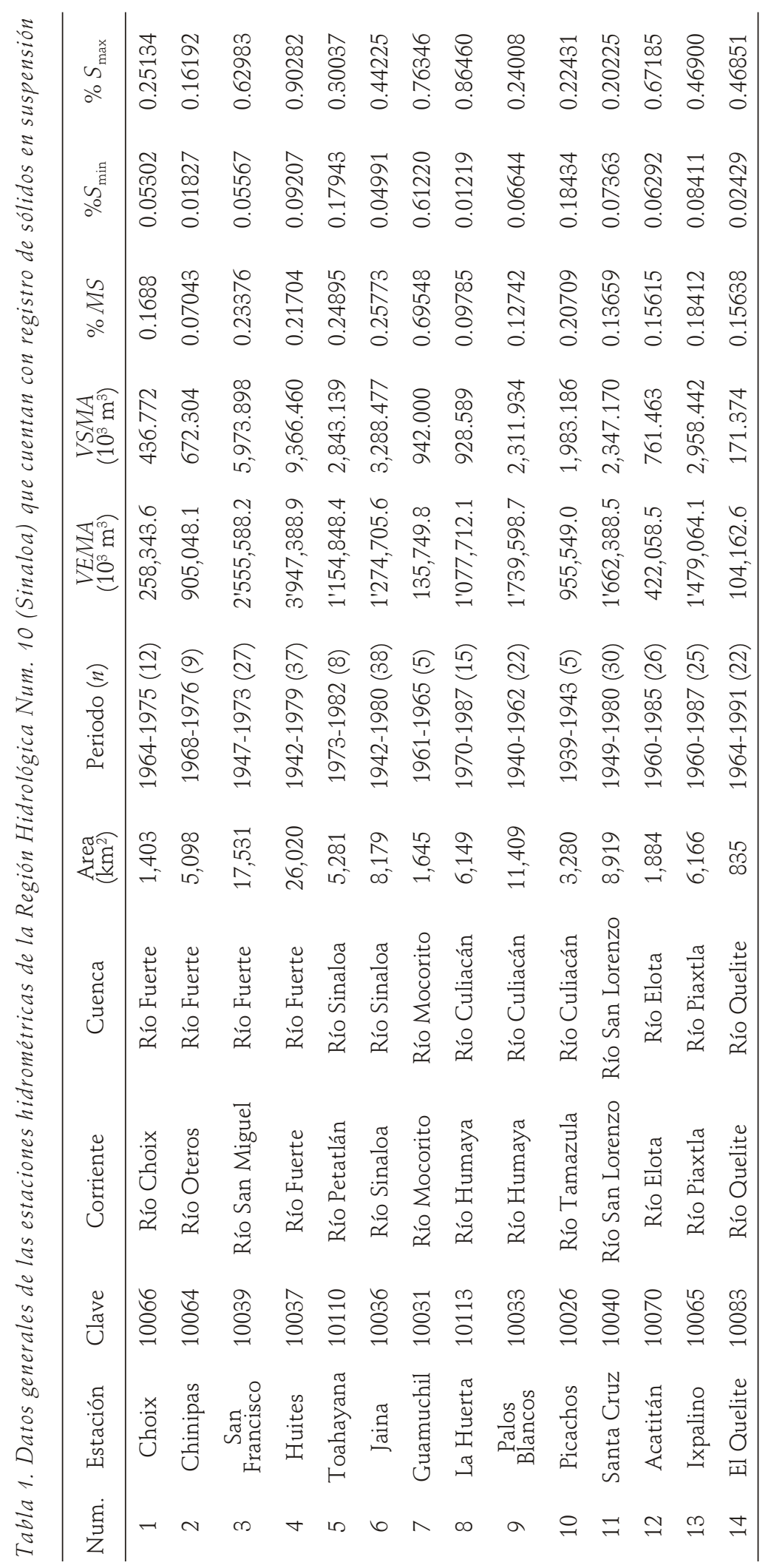

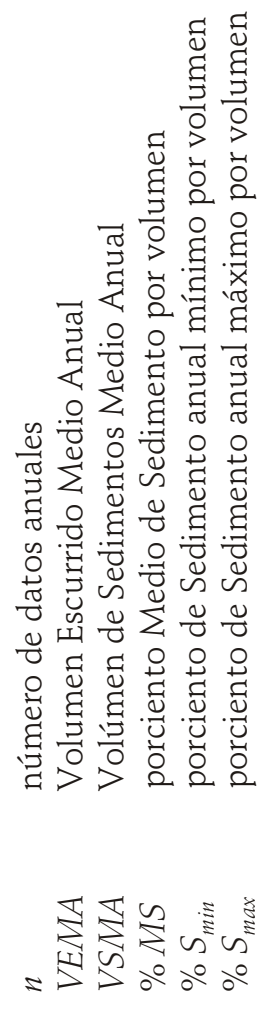


DOI: http://dx.doi.org/10.22201/fi.25940732e.2009.10n3.021

D.F. Campos-Aranda

Tabla 2. Datos anuales de acarreos en suspensión $\left(10^{3} \cdot \mathrm{m}^{3}\right)$ en las nueve estaciones hidrométricas indicadas de la Región Hidrológica Num. 10 (Sinaloa)

\begin{tabular}{|c|c|c|c|c|c|c|c|c|c|}
\hline Num. & El Quelite & Choix & Acatitán & Ixpalino & Jaina & Santa Cruz & $\begin{array}{c}\text { Palos } \\
\text { Balncos }\end{array}$ & $\begin{array}{c}\text { San } \\
\text { Francisco }\end{array}$ & Huites \\
\hline 1 & 141.620 & 655.060 & 580.590 & $1,171.460$ & $4,132.000$ & $1,425.540$ & $1,425.180$ & $2,662.837$ & $8,144.720$ \\
\hline 2 & 236.200 & 568.030 & 879.780 & $2,889.690$ & 678.230 & $2,095.510$ & $2,773.260$ & $1,569.444$ & $53,134.840$ \\
\hline 3 & 24.580 & 519.920 & 647.750 & $1,236.750$ & $1,827.250$ & 860.150 & $2,529.750$ & $13,396.585$ & $4,280.350$ \\
\hline 4 & 38.490 & 567.420 & 831.340 & $2,771.400$ & $2,393.030$ & $1,033.280$ & $2,927.870$ & $2,091.877$ & $2,858.210$ \\
\hline 5 & 120.910 & 542.720 & 260.730 & $1,238.430$ & $2,448.400$ & $1,110.020$ & $1,820.160$ & $1,476.037$ & $3,548.330$ \\
\hline 6 & 113.950 & 244.360 & 168.680 & $1,211.030$ & $3,965.320$ & $1,652.120$ & 710.890 & $4,170.926$ & $3,975.100$ \\
\hline 7 & 170.630 & 358.980 & $1,093.570$ & $3,231.640$ & $8,102 \cdot 160$ & $3,767.100$ & $2,463.700$ & $4,681.901$ & $24,234.430$ \\
\hline 8 & 515.450 & 547.190 & 580.930 & $12,918.480$ & $2,654.120$ & 827.580 & $2,578.910$ & $6,723.194$ & $3,267.030$ \\
\hline 9 & 102.220 & 369.930 & $5,116.340$ & $1,940.270$ & $2,076.430$ & $1,931.220$ & $2,759.850$ & $9,752.386$ & $2,072.750$ \\
\hline 10 & 109.610 & 343.350 & 269.490 & $3,284.670$ & $1,522.230$ & $1,706.710$ & $3,190.100$ & $3,734.470$ & $4,180.870$ \\
\hline 11 & 420.180 & 420.110 & 566.510 & $2,123.510$ & $2,113.830$ & $2,972.860$ & 827.600 & $2,845.605$ & $4,954.230$ \\
\hline 12 & 185.520 & 104.190 & 235.230 & $7,126.430$ & $3,144.390$ & $1,293.660$ & 810.550 & $19,583.970$ & $5,311.450$ \\
\hline 13 & 230.610 & & $1,342.700$ & $2,761.320$ & $5,363.750$ & $3,561.220$ & $2,484.670$ & $10,559.608$ & $6,543.200$ \\
\hline 14 & 22.420 & & 365.760 & $3,497.580$ & $1,870.830$ & $1,669.560$ & 960.660 & $24,324.450$ & $2,445.100$ \\
\hline 15 & 72.840 & & 826.100 & $1,433.980$ & $1,377.470$ & $1,312.070$ & $2,831.440$ & $5,554.045$ & $2,982.290$ \\
\hline 16 & 54.490 & & 374.800 & $1,993.000$ & $8,627.910$ & $4,002.870$ & 640.500 & $8,171.561$ & $7,418.330$ \\
\hline 17 & 699.360 & & 439.380 & $1,828.660$ & $3,829.020$ & $2,357.520$ & 610.970 & $6,952.183$ & $10,783.070$ \\
\hline 18 & 6.910 & & 428.340 & $2,222.420$ & $4,978.960$ & $7,697.990$ & $7,869.130$ & $2,081.015$ & $23,044.530$ \\
\hline 19 & 116.990 & & 344.230 & $1,568.330$ & $4,777.010$ & 847.760 & $1,444.970$ & $2,025.528$ & $9,665.280$ \\
\hline 20 & 170.180 & & 197.090 & $3,932.380$ & $2,527.430$ & $2,584.880$ & $4,173.710$ & $6,194.788$ & $5,291.020$ \\
\hline 21 & 199.350 & & 606.740 & $5,221.970$ & $5,212.640$ & $1,665.440$ & $3,627.570$ & $3,639.592$ & $9,783.870$ \\
\hline 22 & 17.720 & & $1,402.820$ & 641.200 & $2,183.850$ & $4,014.660$ & $1,401.100$ & $4,487.512$ & $6,424.660$ \\
\hline 23 & & & 109.370 & $3,464.370$ & $2,445.550$ & $2,786.100$ & & $1,192.388$ & $15,238.240$ \\
\hline 24 & & & 428.650 & $3,151.620$ & $4,958.360$ & $4,462.080$ & & $1,327.094$ & $29,727.550$ \\
\hline 25 & & & 459.420 & $1,100.450$ & $4,201.930$ & $1,045.070$ & & $3,495.104$ & $10,625.470$ \\
\hline 26 & & & $1,241.700$ & & $3,543.460$ & $1,627.650$ & & $6,464.559$ & $15,214.850$ \\
\hline 27 & & & & & 990.580 & $2,403.780$ & & $2,136.589$ & $3,686.490$ \\
\hline 28 & & & & & $2,656.510$ & $2,572.000$ & & & $4,874.130$ \\
\hline 29 & & & & & $4,687.220$ & $3,038.830$ & & & $9,486.190$ \\
\hline 30 & & & & & $4,214.290$ & $2,089.680$ & & & $9,075.720$ \\
\hline 31 & & & & & $3,561.800$ & & & & $11,181.060$ \\
\hline 32 & & & & & $4,744.300$ & & & & $7,671.690$ \\
\hline 33 & & & & & $1,150.420$ & & & & $5,070.060$ \\
\hline 34 & & & & & $3,513.130$ & & & & $3,551.330$ \\
\hline 35 & & & & & $2,385.630$ & & & & $3,034.000$ \\
\hline 36 & & & & & $2,346.520$ & & & & $6,391.460$ \\
\hline 37 & & & & & $2,968.640$ & & & & $7,387.120$ \\
\hline 38 & & & & & 787.530 & & & & \\
\hline
\end{tabular}




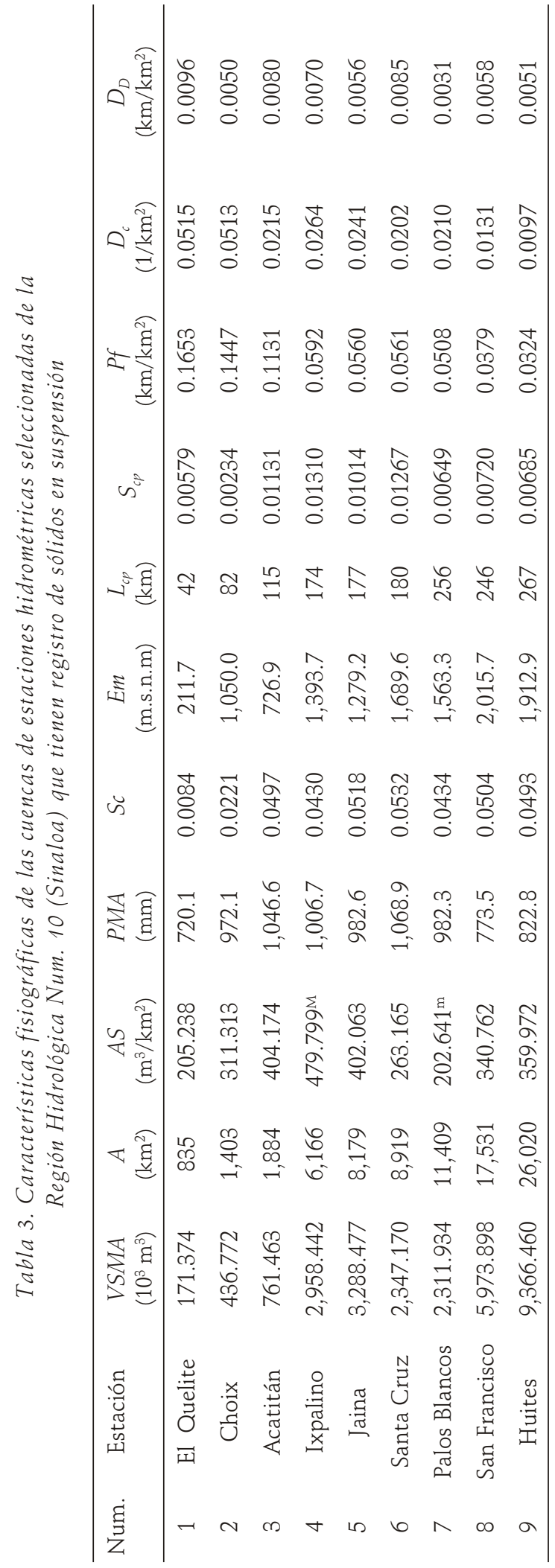

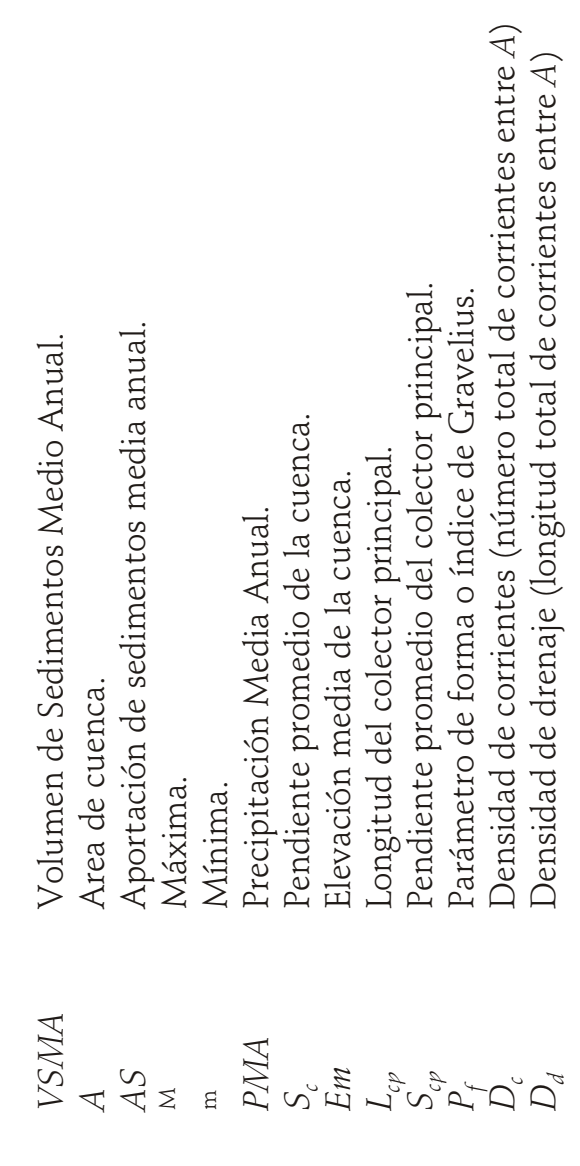




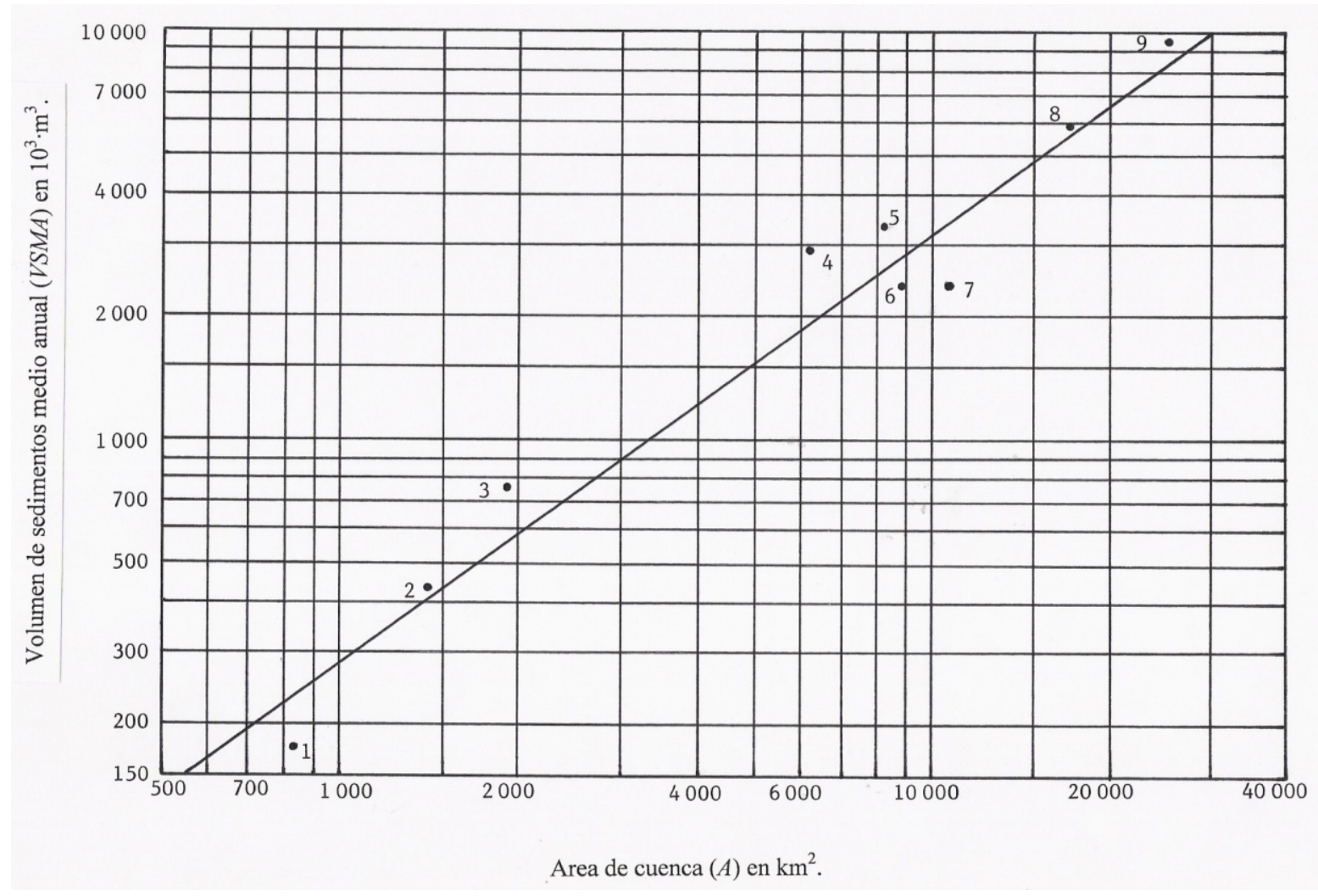

Figura 1. Relación entre el volumen de sedimentos medio anual (VSMA) en miles de $m^{3}$ y el área de cuenca (A) en $\mathrm{km}^{2}$ en la Región Hidrológica Num. 10 (Sinaloa)

Tabla 4. Predicciones adimensionales asociadas a las probabilidades indicadas obtenidas con el método regional citado

\begin{tabular}{lccccc}
\hline Método regional: & \multicolumn{5}{c}{ Probabilidad de no excedencia en \% } \\
& 50 & 66.7 & 75 & 80 & 90 \\
\hline Estaciones - años & 0.782 & 1.042 & 1.229 & 1.379 & 1.885 \\
MPP ponderados & 0.808 & 1.061 & 1.239 & 1.380 & 1.847 \\
\hline
\end{tabular}

$$
\begin{aligned}
& b_{0}=\bar{x}=\sum_{i=1}^{n} x_{i} \\
& b_{1}=\sum_{i=1}^{n-1} \frac{(n-i) x_{i}}{n(n-1)} \\
& b_{2}=\sum_{i=1}^{n-2} \frac{(n-i)(n-i-1) x_{i}}{n(n-1)(n-2)},
\end{aligned}
$$

con base en tales valores, se buscaron relaciones lineales $b_{i+1}=\xi+m \cdot b_{i}$ y se obtuvieron:

$$
b_{1}=-80.64551+0.72831 \cdot b_{0}
$$

con

$$
\begin{aligned}
& R^{2}=0.9982 \quad E E E=97.1 \text { miles de } \mathrm{m}^{3} \\
& b_{2}=-47.53955+0.82385 \cdot b_{1}
\end{aligned}
$$

con

$$
R^{2}=0.9993 \quad E E E=50.4 \text { miles de } \mathrm{m}^{3} .
$$



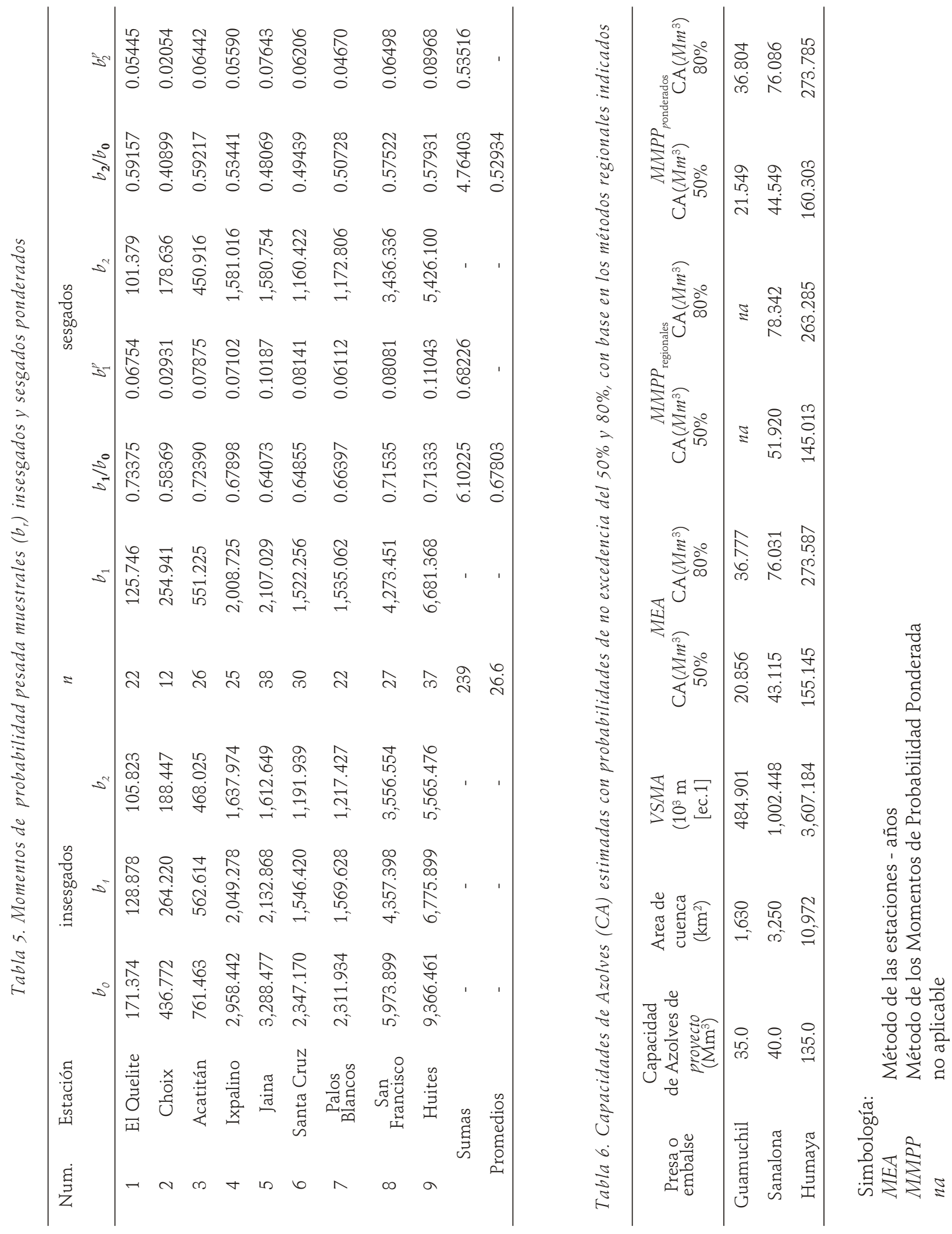
DOI: http://dx.doi.org/10.22201/fi.25940732e.2009.10n3.021

D.F. Campos-Aranda

Entonces, a partir de la ecuación 1 se estima $V S M A=b_{0}$ y con las ecuaciones 7 y 8 se obtienen $b_{1}$ y $b_{2}$ para calcular los momentos $\mathrm{L}$ y hacer el ajuste de la distribución GVE (Stedinger et al., 1993; Campos, 2006), con la cual se obtienen las predicciones $X$, o volúmenes de sedimentos en suspensión $\left(10^{3} \cdot \mathrm{m}^{3}\right)$ asociados a las probabilidades de no excedencia citadas.

\section{Método de los MPP ponderados}

Los valores de los estimadores sesgados de los MPP $\left(b_{r}^{*}\right)$ se obtienen a partir del registro ordenado en magnitudes decrecientes $\left(x_{i}\right)$ con la expresión siguiente (Stedinger et al., 1993):

$$
b_{r}^{*}=\frac{1}{n} \sum_{i=1}^{n} x_{i}\left[1-\frac{(i-0.35)}{n}\right]^{r}
$$

después, se estandarizan dividiéndolos entre $b_{0}$ (media aritmética) y posteriormente se ponderan con base en la amplitud $\left(n_{l}\right)$ de cada registro del cual proceden $(l=1,2$, $\ldots, 9)$ esto es:

$$
\left(b_{r}^{*}\right)^{p}=\frac{b_{r}^{*}}{b_{0}}\left(n_{l} / \Sigma\right)
$$

$\Sigma$ es su suma, es decir 239. También en la tabla 5 se presentan los MPP sesgados estandarizados y su ponderación. Con base en los valores $\left(b_{0}^{*}\right)^{p}=1.00000$,

$\left(b_{1}^{*}\right)^{p}=0.68226$ y $\left(b_{2}^{*}\right)^{p}=0.53516$ se ajusta la distribución GVE por momentos L (Stedinger et al., 1993; Campos, 2006) para obtener: $u=0.65257$,

$a=0.40797$ y $k=-0.22462$, así como las predicciones adimensionales $(X)$ citadas en la tabla 4 . El procedimiento de aplicación es idéntico al del método de las estaciones-años.

\section{Otros métodos regionales}

Los procedimientos regionales basados en un periodo común de datos (Campos, 2006), no fueron aplicables debido a la discrepancia en el inicio y final de cada registro de datos (tabla 1).

\section{Aplicaciones numéricas}

Del Razo (1999) indica que las presas Eustaquio Buelna (Guamuchil), Adolfo López Mateos (Humaya) y Sanalona de la Región Hidrológica No. 10, requieren modificaciones; por ello se revisarán sus valores de proyecto en cuanto a capacidad de azolves, aceptando que su vida útil (VU) sea de 50 años y que por transporte de fondo se incrementará un $10 \%$. Entonces la capacidad de azolves $(C A)$ en $\mathrm{Mm}^{3}$ (millones de $\mathrm{m}^{3}$ ) será igual a:

$$
C A=1.10 \cdot X \cdot V U
$$

en la cual, $X$ es la predicción del volumen de sedimentos anual en $\mathrm{Mm}^{3}$ con la probabilidad de no excedencia seleccionada. Por ejemplo, para la Presa Guamuchil con un área de cuenca de $1,630 \mathrm{~km}^{2}$, la ecuación 1 conduce a un valor del VSMA $=484.901$ miles de $\mathrm{m}^{3}$, de manera que el método de las estaciones-años con predicciones adimensionales de 0.782 y 1.379 para el 50 y $80 \%$ de probabilidad de no excedencia conduce a valores de $X$ igual a 379.193 y 668.678 miles de $\mathrm{m}^{3}$, para tales probabilidades. Entonces la capacidad de azolves según práctica actual usando la mediana y el enfoque propuesto serán:

$$
\begin{aligned}
& C A=1.10 \cdot(379.193 / 1,000) \cdot 50=20.856 \mathrm{Mm}^{3} \\
& C A=1.10 \cdot(668.678 / 1,000) \cdot 50=36.777 \mathrm{Mm}^{3}
\end{aligned}
$$

En la tabla 6 se presentan los datos y resultados obtenidos con los tres métodos regionales expuestos. Se concluye que las capacidades de azolves de proyecto corresponden al orden de magnitud cuando se emplea la mediana, con un valor sobrado en la presa Guamuchil y uno escaso en Humaya. Sin embargo, cuando se utiliza la probabilidad de no excedencia del $80 \%$, únicamente en la presa Guamuchil su estimación de CA está correcta y en las otras dos es bastante reducida, llegando solamente al 52 y $50 \%$ en Sanalona y Humaya.

\section{Conclusiones}

Se han expuesto los procedimientos de los métodos regionales que fueron susceptibles de aplicar en los nueve registros seleccionados de acarreos en suspensión, disponibles en la Región Hidrológica No. 10 para la estimación en cuencas sin aforos de predicciones de diseño con menor riesgo de ser excedidas, que las comúnmente utilizadas, correspondientes a la media aritmética o la mediana.

La similitud numérica de los resultados mostrados en la tabla 6 para las estimaciones de la capacidad de azolves, destacan la confiabilidad de los métodos regionales.

Se recomienda continuar este tipo de análisis en otras regiones hidrológicas o geográficas, para disponer de mejores técnicas de estimación de valores de diseño del volumen de acarreos en suspensión, en este caso, 
DOI: http://dx.doi.org/10.22201/fi.25940732e.2009.10n3.021

Estimación probabilística del volumen de acarreos en suspensión en cuencas sin aforos de la Región Hidrológica No. 10 (Sinaloa)

con menor riesgo de ser excedidos y calcular con ello, capacidades de azolves más confiables.

\section{Referencias}

Campos-Aranda D.F. Aplicación del método del índice de crecientes en la región hidrológica número 10, Sinaloa. Ingeniería Hidráulica en México, IX(3):41-55, septiembrediciembre de 1994.

Campos-Aranda D.F. Predicciones de volúmenes de sólidos en suspensión en cuencas sin aforos en la Región Hidrológica No. 25 (San Fernando-Soto La Marina). Tlaloc, (33):22-28, enero-abril del 2005.

Campos-Aranda D.F. Análisis probabilístico univariado de datos hidrológicos. Tema 6.6: Distribución General de Valores Extremos, pp. 99-102, Cap. 8: Análisis probabilístico con métodos regionales, pp. 133-161. Avances en Hidráulica 13. AMH-IMTA. México, DF. 2006. 172 p.

Cunnane, C. Methods and Merits of Regional Flood Frequency Analysis. Journal of Hydrology, 100:269-290. 1988.

Del Razo V. Rehabilitación y/o modificación de presas, pp. 43-62. En: El desarrollo de las presas en México, Editores Arreguín F.I., Herrera C., Marengo H. y Paz Soldán G.A. Avances en Hidráulica 5. AMH-IMTA. México DF. 1999. $224 \mathrm{p}$.

Domínguez E.J.Y. Selección de estaciones hidrométricas homogéneas de una región hidrológica. XIV Congreso
Nacional de Hidráulica. Tomo I, ponencia 20. Acapulco, Guerrero. Octubre de 1996.

Escalante S.C. Multivariate Extreme Value Distribution with Mixed Gumbel Marginals. Journal of the American Water Resources Association, 34(2):321-333, 1998.

Escalante S.C. y Reyes Chávez L. Técnicas estadísticas en hidrología. Apéndice A, pp. 291-298. Facultad de Ingeniería de la UNAM. México DF. 2002. 298 p.

Instituto Mexicano de Tecnología del Agua (IMTA). Banco Nacional de Datos de Aguas Superficiales (BANDAS). 8 CD's. Volumen 1. Región III: Pacífico Norte. CNA-SEMARNAT. Jiutepec, Morelos. 2003.

Morris, G.L., Fan J. Reservoir Sedimentation Handbook. Chapter 5: Sediment properties, pp.5.1-5.28 and chapter 7: Sediment yield from watersheds, pp 7.1-7.44. McGraw-Hill Companies, Inc. New York, USA. 1998.

Secretaría de Recursos Hidráulicos (SRH). Actualización al Boletin Hidrológico Num. 36. (Región Hidrológica Num. 10. Sinaloa. Tomo VI. Periodo 1970-1973). Subsecretaría de Planeación. México DF. 1975.

Stedinger J.R., Vogel R.M., Foufoula-Georgiou E. Frequency Analysis of Extreme Events. Chapter 18, pp. 18.1-18.66 in Handbook of Hydrology, editor in chief David R. Maidment. McGraw-Hill, Inc. New York, USA. 1993.

Varas E. Estimación de momentos ponderados regionales de caudales máximos diarios. Ingeniería Hidráulica en México. XV(1):51-61, enero-abril de 2000.

\section{Semblanza del autor}

Daniel Francisco Campos-Aranda. Es ingeniero civil con maestría y doctorado en ingeniería por la División de Estudios de Posgrado de la Facultad de Ingeniería de la UNAM. Inició su actividad profesional en la Secretaría de Recursos Hidráulicos en junio de 1972 y la concluyó en diciembre de 1990 en la Comisión Nacional del Agua. Ha publicado 31 artículos en la revista Ingeniería Hidráulica en México, 9 en Agrociencia y 9 en Tláloc, es autor del texto Introducción a los Métodos Numéricos, publicado por la Editorial Universitaria Potosina en 2003 y de los libros: Agroclimatología Cuantitativa de Cultivos, publicado por Editorial Trillas en 2005 y Análisis Probabilístico Univariado de Datos Hidrológicos publicado por la AMH-IMTA en 2006. En enero del 2007, publicó el texto Estimación y Aprovechamiento del Escurrimiento. Fue investigador nacional (nivel I: expediente 7273) desde julio de 1991 hasta el diciembre del 2007. Desde el 2003 es profesor Jubilado de la Universidad Autónoma de San Luis Potosí. En 2008 recibió el premio nacional "Francisco Torres H." que otorga la $\mathrm{AMH}$. 